\title{
CARBONO ORGÁNICO, NITRÓGENO Y DENSIDAD APARENTE EN SUELOS DE FINCAS CON GANADERÍA BOVINA DE CRÍA
}

\section{RESUMEN}

\section{Carbono orgánico, nitrógeno y densidad aparente en suelos de fincas con ganadería bovina de} cría. Las pasturas tienen un alto potencial de remoción de carbono cuando son manejadas adecuadamente. En Costa Rica, hay poca información sobre los contenidos de carbono (C) y nitrógeno (N) del suelo, así como sobre el nivel de compactación con respecto a la vegetación natural en las pasturas. Entre febrero 2015 y abril 2017, se realizaron muestreos de suelos a $10 \mathrm{~cm}$ de profundidad para observar la concentración de C y N, la Masa de Carbono Orgánico del Suelo (COS) y Nitrógeno Total $\left(\mathrm{N}_{t}\right)$, así como la Densidad Aparente (Da) entre 0 y $10 \mathrm{~cm}$ de profundidad en 38 fincas de ganadería bovina de cría, distribuidas en todo el país con diferentes grados de pendiente. No se observaron diferencias para las concentraciones de $\mathrm{C}$ y $\mathrm{N}$ en bosque en relación con la pendiente. Aunque en suelos con pasturas se observaron diferencias con promedios de 1,96, 1,40 y 1,32 \% $\mathrm{C}$ para pendientes planas, onduladas y quebradas respectivamente. La concentración de $\mathrm{N}$ y la Da mostraron diferencias entre bosque $\left(0,38 \%\right.$ y $\left.1,02 \mathrm{~g} \cdot \mathrm{cm}^{-3}\right)$ y pastura $\left(0,27 \%\right.$ y $\left.0,93 \mathrm{~g} \cdot \mathrm{cm}^{-3}\right)$. Se concluye que los contenidos de COS son semejantes en pasturas y bosque, donde los contenidos de $\mathrm{N}$ y la Da influyen de manera importante en la relación carbono: nitrógeno (C:N) de la pastura.

Palabras clave: pasturas tropicales, densidad aparente, carbono del suelo.

\section{INTRODUCCIÓN}

En Costa Rica, normalmente las remociones de carbono atmosférico se asocian al crecimiento de árboles, en bloques forestales que por lo general son de una misma especie exótica y al crecimiento sucesional en su estado de bosque secundario. No obstante, algunas experiencias de remoción de carbono en fincas ganaderas sugieren que el suelo, bajo un manejo de pasturas adecuado, sería la mejor fuente de remoción de C de estos sistemas de producción (Abarca 2016). Estudios recientes indican que a nivel global, el solo reducir las emisiones de Gases con Efecto Invernadero (GEl), bajo los esquemas de negocios de la vida moderna actual no es viable, se requiere una remoción extensa de carbono atmosférico a corto plazo para mantener el calentamiento global a niveles menores

1 Instituto Nacional de Innovación y Transferencia en Tecnología Agropecuaria, INTA. Costa Rica. sabarca@inta.go.cr, rsoto@inta.go.cr, farguedas@inta.go.cr 
de $2{ }^{\circ} \mathrm{C}$ según lo previsto en el Acuerdo de París. La mejor alternativa, aunque insuficiente, incluye la captura de carbono en los sistemas agrícolas mediante la fertilización nitrogenada y la intensificación del riego (Boysen et al. 2017).

Por otra parte, FAO (2002), indica que hay más cantidad de $\mathrm{C}$ en el primer metro de suelo, que en toda la biomasa terrestre y que este puede acumularse o mineralizarse de acuerdo a su manejo. En los suelos, se encuentran tanto las formas orgánicas como las inorgánicas del C; lo habitual es que el uso y la gestión de la tierra (manejo) tengan un impacto mayor sobre los depósitos de $\mathrm{C}$ orgánico. En consecuencia, los métodos que se utilizan en las determinaciones de $\mathrm{C}$ en suelos, son dirigidos al $\mathrm{C}$ orgánico (IPCC 2006). El manejo de las coberturas del suelo tiene incidencia en los contenidos de COS a través de los cambios en la existencia de C, producto de los aportes de la materia orgánica. Se ha observado que los suelos convertidos a pasturas perennes bien manejadas, incrementan los contenidos del carbono orgánico (Guo y Gifford 2002). En sistemas silvopastoriles de España Scott et al (2011), encontraron cantidades de COS entre 20,0 a 41,2 t.ha-1, en los primeros $25 \mathrm{~cm}$ de profundidad, donde los agregados de 250 a $2000 \mu \mathrm{m}$ fueron los que retuvieron más carbono.

En la zona tropical muy húmeda de Costa Rica, se han encontrado diferencias en los contenidos de carbono orgánico en el perfil del suelo, entre pasturas nativizadas (Axonupus) y mejoradas (Brachiaria sp.); así como entre órdenes de suelo contrastantes (Andisol e Inceptisol) (Veldkamp 1994). Más recientemente, en la misma zona de vida, se ha observado en pasturas de Cayman ${ }^{\circledR}$, que el pastoreo con periodos adecuados de rebrote (42-45 días) y de ocupación cortos (2-3 días máximo), incrementaron rápidamente los contenidos de C (Hernández et al. 2014). A pesar del potencial de remoción de $C$ en suelos bajo pasturas, un sondeo realizado en el 2013 (INTA-CORFOGA 2014), mostró que el 81\% de las muestras tomadas en pasturas, presentaron contenidos bajos de carbono en suelo.

Recientemente, Costa Rica ha inscrito ante la Convención de Cambio Climático de Naciones Unidas, la Acción de Mitigación Nacionalmente Apropiada (NAMA) en ganadería bovina (Costa Rica 2015). Observando un alineamiento con la carbono neutralidad que ha sido la forma costarricense de realizar la mitigación; entendida bajo la definición de la norma INTE 12-01-06:2011 (INTECO 2011), que permite relacionar las remociones de carbono y emisiones de GEl de las organizaciones productivas para establecer un resultado neto en Dióxido de Carbono Equivalente $\left(\mathrm{CO}_{2} \mathrm{e}\right)$, en este caso en los terrenos de fincas ganaderas, donde hay bosques, árboles dispersos y suelos gestionados con gran potencial de retener carbono, estableciendo un balance entre las emisiones de GEl y las remociones de carbono.

\section{MATERIALES Y MÉTODOS}

Se seleccionaron 38 fincas ganaderas de cría de ganado bovino, distribuidas en 19 cantones rurales de las siete provincias de Costa Rica, cuyos suelos pertenecen a los órdenes: Inceptisol (18), Ultisol (12), Andisol (4) y Alfisol (4). En todas las fincas se estudiaron dos coberturas de suelo (bosque y pasto), con diferentes topografías, altitudes y regímenes de precipitación; valoradas por técnicos de la Corporación de Fomento Ganadero (CORFOGA), el Ministerio de Agricultura y Ganadería (MAG) y el Instituto Nacional de Innovación y Transferencia en Tecnología Agropecuaria (INTA); con un manejo adecuado de pasturas dentro del entorno de cada sitio. En las mismas se tomaron muestras de suelo para la determinación de la concentración de $\mathrm{C}$ y $\mathrm{N}$, la masa de carbono orgánico del suelo (COS), el $\mathrm{N}_{\mathrm{t}}$, y la Da. 
Cuadro 1. Altitud, precipitación y topografía de las fincas evaluadas. Costa Rica. Febrero 2015 y abril 2017.

\begin{tabular}{|c|c|c|c|c|c|}
\hline \multirow{2}{*}{ Altitud (msnm) } & \multirow{2}{*}{ Precipitación (mm) } & \multicolumn{3}{|c|}{ Topografía } & \multirow{2}{*}{ Total } \\
\hline & & Plana & Ondulada & Quebrada & \\
\hline \multirow{6}{*}{$0-500$} & $1500-2000$ & 1 & & 2 & 3 \\
\hline & $2000-2500$ & 4 & & 1 & 5 \\
\hline & $2500-3000$ & & 1 & 1 & 2 \\
\hline & $3000-3500$ & 5 & & & 5 \\
\hline & $3500-4000$ & 1 & 3 & 1 & 5 \\
\hline & $4000-4500$ & 2 & 1 & & 3 \\
\hline \multirow{4}{*}{$500-1000$} & $2000-2500$ & & 1 & & 1 \\
\hline & $2500-3000$ & & 3 & 2 & 5 \\
\hline & $3500-4000$ & & 2 & 3 & 5 \\
\hline & $>4500$ & 1 & 1 & & 2 \\
\hline $1000-1500$ & $2000-2500$ & & & 1 & 1 \\
\hline $1500-2000$ & $2500-3000$ & & & 1 & 1 \\
\hline \multicolumn{2}{|c|}{ Total de fincas } & 14 & 12 & 12 & 38 \\
\hline
\end{tabular}

Los muestreos se realizaron siempre en la época de menor precipitación, entre febrero 2015 y abril 2017. Dado que las fincas tenían diferente grado de pendiente, se procedió a valorar la topografía presente utilizando el cuadro 2 , donde se indican los rangos de pendiente normalmente utilizados por los ganaderos para uso del pastoreo, con un sentido más fisiográfico de acuerdo con ecólogos del calibre de Odum, Barret, Holdridge y Tosi.

Cuadro 2. Escala de pendientes para evaluar la topografía en fincas ganaderas. Costa Rica. Febrero 2015 y abril 2017.

\begin{tabular}{|c|c|}
\hline Tipo & Pendiente \% \\
\hline Plano & $0-10$ \\
\hline Ondulado & $10-25$ \\
\hline Quebrado & $>25$ \\
\hline
\end{tabular}

Adaptado de: INTA CORFOGA 2013 y Abarca 2018.

Las muestras para la determinación de la concentración de $\mathrm{C}$ y $\mathrm{N}$, se tomaron con barreno a $20 \mathrm{~cm}$ de profundidad. Los análisis de laboratorio se realizaron en el laboratorio de suelos del INTA y de la Universidad de Costa Rica. Para la estimación de la Da se utilizó la técnica del cilindro, de acuerdo con la metodología descrita por Alvarado y Forsythe
2005, utilizando cilindros de $5 \mathrm{~cm}$ de largo y $5 \mathrm{~cm}$ de diámetro en forma vertical, después de remover la hojarasca. Para la determinación del C y N, se utilizó el método de Dumas (combustión).

\section{Estimación de la cantidad de COS}

Para la estimación del carbono de suelo por hectárea, en una lámina de $10 \mathrm{~cm}$ de suelo, se siguió el procedimiento establecido por Veldkamp (1994), para suelos y zonas de vida tropicales, de acuerdo a la siguiente ecuación:

$$
C O S=C s{ }^{*} L s^{*} D a^{*} 104
$$

Donde:

$$
\begin{aligned}
& \cos =\text { Carbono Orgánico Suelo (t.ha-1) } \\
& \text { Cs = Contenido de carbono en el suelo (g.g) } \\
& \text { Ls = Lámina de suelo }(\mathrm{cm}) \\
& \mathrm{Da} \quad=\text { Densidad Aparente }\left(\mathrm{g} . \mathrm{cm}^{-3}\right)
\end{aligned}
$$

Para obtener el dióxido carbono equivalente $\left(\mathrm{CO}_{2}\right.$ e), se utilizó la conversión en función de los pesos moleculares del $\mathrm{CO}_{2}$ y el C (44/12). 


\section{RESULTADOS Y DISCUSIÓN}

La concentración de C no varió significativamente entre los suelos cubiertos con bosque secundario y pastura, el promedio fue de 1,61 \%. En relación con la topografía, no se obtuvieron diferencias en el bosque, aunque se observó una tendencia a la reducción en los sitios quebrados, sin embargo, las pasturas en sitios planos $(1,96 \pm 1.71 \%)$ presentaron diferencias $(p<0,1)$ con respecto a lugares ondulados $(1,40 \pm 0.45)$ y quebrados $(1,32 \pm 44)$; (ver Cuadro 2). La concentración de $\mathrm{N}$ fue significativamente mayor $(p<0,05)$ en el bosque que en la pastura y no presentó variación en relación con la topografía para ninguna de las dos coberturas del suelo. Con respecto a la relación C: N, no se obtuvieron diferencias entre el bosque y las pasturas, ni en topografía dentro de las coberturas. Sin embargo, se observó una tendencia hacia el incremento en la pastura, para las tres topografías descritas. La cantidad de COS a $10 \mathrm{~cm}$ de profundidad presentó diferencias significativas $(p<0001)$ entre coberturas y no con respecto a la topografía dentro de las coberturas. La Da varió significativamente ( $p>0001)$ entre coberturas; el bosque presentó diferencias en relación con la topografía y los sitios ondulados y planos, tuvieron la menor compactación $(0,82 \pm 0,24$ y $0,96 \pm 0,15 \mathrm{~g} . \mathrm{cm}^{-3}$ respectivamente) mostrando diferencia $(p>0,05)$ con los sitios quebrados $\left(1,07 \pm 0,14 \mathrm{~g} \cdot \mathrm{cm}^{-3}\right)$.

Trabajos realizados por Oliva et al 2017, reportan contenidos más altos (3,29\%) de C que los observados en este trabajo, en sistemas silvopastoriles con Pinus patula y forrajes nativos de Perú. En Costa Rica, Weintraub et al 2016, trabajando en los bosques de la parte sur del lado del Pacífico de la cordillera de Talamanca, observó que a medida que se sube en la cordillera, se incrementan las concentraciones de $\mathrm{C}$ y la relación $\mathrm{C}: \mathrm{N}$; el $\mathrm{N}$ también se incrementa pero más levemente que el carbono. Así mismo, la relación C:N declina conforme aumentan la temperatura y la precipitación, variables climáticas que aumentan conforme se desciende en altura sobre el nivel del mar, en la región donde se realizó la investigación. En relación a la densidad aparente, Scott et al 2011 en sistemas silvopastoriles de Extremadura en España, a una profundidad entre $0-25 \mathrm{~cm}$ en suelos con $60 \%$ de limo, reporta valores de $1,30 \mathrm{~g} \mathrm{~cm}^{-3}$, siendo más altos que los observados en esta investigación para pasturas. No obstante, Hernández et al 2014, bajo condiciones de trópico muy húmedo de Costa Rica en pasturas mejoradas y bien manejadas, obtuvieron valores de C, Da y COS concordantes con los obtenidos en este estudio. Así mismo, Ibrahim et al 2006, en fincas ganaderas de Costa Rica y Nicaragua, tampoco observaron diferencias entre el bosque secundario y las pasturas con especies mejoradas.

Posiblemente la cantidad de COS no mostró diferencias entre coberturas, debido a que la Da fue mayor en el suelo con pastura y la concentración de C, aunque no estadísticamente significativa, fue lo suficientemente mayor en el bosque para igualar los valores promedio. En concordancia con lo anterior, en la pastura se obtuvo una correlación negativa $(-0,85)$ altamente significativa $(p<0001)$, indicando que una menor concentración de $\mathrm{N}$ produjo una relación C:N mayor, lo que sugiere una menor actividad biológica con respecto al bosque, favoreciendo el almacén de $\mathrm{C}$ en suelos con pasturas entre $0-10 \mathrm{~cm}$ de profundidad.

Cuadro 3. Concentración de C, N, Relación C:N y cantidad de COS; en suelos de fincas de ganadería bovina de cría en Costa Rica. Febrero 2015 y abril 2017.

\begin{tabular}{|c|c|c|c|c|}
\hline Variable & Cobertura & Promedio & LI & LS \\
\hline \multirow{2}{*}{ Carbono (\%) } & Bosque & $1,74 \mathrm{a}$ & 1,50 & 1,97 \\
\hline & Pastura & $1,67 \mathrm{a}$ & 1,46 & 1,87 \\
\hline \multirow{2}{*}{ Nitrógeno (\%) } & Bosque & $0,38 \mathrm{a}$ & 0,27 & 0,49 \\
\hline & Pastura & $0,27 \mathrm{~b}$ & 0,23 & 0,32 \\
\hline \multirow{2}{*}{ Relación C:N } & Bosque & $5,76 \mathrm{a}$ & 4,48 & 7,04 \\
\hline & Pastura & $6,83 \mathrm{a}$ & 5,77 & 7,89 \\
\hline \multirow{2}{*}{$\cos ($ t.ha-1) } & Bosque & $15,9 a$ & 14,0 & 17,8 \\
\hline & Pastura & $16,6 \mathrm{a}$ & 14,6 & 17,8 \\
\hline \multirow{2}{*}{$\begin{array}{l}\text { D. Aparente } \\
\left(\mathrm{g} \cdot \mathrm{cm}^{-3}\right)\end{array}$} & Bosque & $0,93 a$ & 0,85 & 1,02 \\
\hline & Pastura & $1,02 \mathrm{~b}$ & 0,98 & 1,05 \\
\hline
\end{tabular}

Promedios con letra común dentro de una misma variable no son significativamente diferentes ( $p>0,05)$. Limites Inferior y Superior del intervalo de confianza a $0,95 \%$ de la media. 
En conclusión, las diferencias entre bosque y pastura para las variables estudiadas, se obtuvieron básicamente en la concentración de $\mathrm{N}$ y la Da. Aunque no se obtuvieron diferencias significativas en la relación C:N, se observó una tendencia a ser mayor en la pastura, lo que sugiere un potencial para retener más carbono que el bosque; no obstante, podría ser la mayor limitante para el incremento de productividad de las especies forrajeras que componen las pasturas de este tipo de ganadería

\section{LITERATURA CITADA}

Abarca, S. 2016. Emisión de gases de efecto invernadero y absorción de carbono en fincas ganaderas. Revista Alcances Tecnológicos 11(1):71-73.

Abarca, S. 2018. Capacidad de carga de la finca ganadera bovina. Revista Universidad Técnica Nacional. 20 (81):22-25.

Alvarado, A; Forsythe, W. 2005. Variación de la densidad aparente en órdenes de suelos de Costa Rica (en línea). Agronomía Costarricense 29(1):85-94.

MAG (Ministerio de Agricultura y Ganadería). 2015. NAMA Ganadería. San José, Costa Rica. 27p.

Boysen, L; Lucht, W; Gerten, D; Heck, V; Lenton, T; Joachim Schellnhuber, H. 2017. The limits to global-warming mitigation by terrestrial carbon removal (en línea). Earth's Future Journal 5 (5):417-536. Consultado 5 may. 2017. Disponible en http://onlinelibrary.wiley.com/doi/10.1002/2016EF000469/full

FAO (Organización de las Naciones Unidas para la Agricultura y la Alimentación). 2002. Captura de carbono en los suelos para un mejor manejo de la tierra (en línea). Informe sobre recursos mundiales de suelos. $N^{\circ}$ 96. ISSN 1020-430-X. 61 p. Consultado 18 abr. 2018. Disponible en http://www.fao.org/docrep/005/ Y2779S/y2779s00.htm

Guo, L.; Gifford, R. 2002. Soil carbon stocks and land use change: a meta-analysis. Global Change Biology 8(4):345-360.

Hernández, M; Abarca, S; Soto, R. 2014. Evaluación de pasto Brachiaria híbrido cv Cayman, en pastoreo en el trópico muy húmedo de Costa Rica. Revista Universidad Técnica Nacional 16(70):48-54.

INTECO (Instituto de Normas Técnicas de Costa Rica). 2016. Gases de efecto invernadero. Parte 1: especificación con orientación, a nivel de las organizaciones, para la cuantificación y el informe de las emisiones y remociones de gases de efecto invernadero. Sistema de gestión para demostrar la C-Neutralidad.
Requisitos. Norma: INTE/ISO 14064-1:2006. San José, CR. 33p.

INTA (Instituto Nacional de Innovación y Transferencia en Tecnología Agropecuaria); CORFOGA (Corporación de Fomento Ganadero). 2014. Informe 2013. valoración servicios ecosistémicos en fincas ganaderas de cría. San José, Costa Rica. 21p.

IPCC (Grupo Intergubernamental de Expertos sobre el Cambio Climático). 2006. Pastizales (en línea). In: Directrices para los inventarios nacionales de gases efecto invernadero. Agricultura, silvicultura y otros usos de la Tierra. Capítulo 6. Consultado 18 abr. 2018. Disponible en http://www.ipccnggip.iges.or.jp/ public/2006gl/spanish/pdf/4_Volume4/V4_06_Ch6_ Grassland.pdf

Oliva, M.; Culqui, L.; Leiva, S.; Collazos, R.; Salas, R.; Vásquez, H.; Maicelo, J. 2017. Reserva de carbono en un sistema silvopastoril compuesto de Pinus patula y herbáceas nativa (en línea). Scientia Agropecuaria 8(2):149 - 157. Consultado 18 abr. 2018. Disponible en http://revistas.unitru.edu.pe/index.php/scientiaagrop

Scott, D; Moreno, G; Mosquera M. R; Nair, K.P Nair, V. 2011. Soil carbon storage as influenced by tree cover in the Dehesa cork oak silvopasture of central-western Spain. (en línea). Journal of Environmental Monitoring 13:1897. Consultado 18 abr. 2018. Disponible en http://pubs.rsc.org/en/Content/ArticleLanding/2011/ EM/c1em10059a\#!divAbstract

Veldkamp, E. 1994. Organic carbon turnover in three tropical soil under pasture after deforestation. In: soil organic carbon dynamics in pastures established after deforestation in the humid tropic of Costa Rica. Tesis Ph.D. Wageningen, Holanda, Wageningen University. $117 \mathrm{p}$.

Weintraub, S; Cole, R; Schmitt, C; All, J. 2016. Climatic controls on the isotopic composition and availability of soil nitrogen across mountainous tropical forest. Ecosphere 7(8):1-13. 
